\title{
In-Situ laser fenestration of endovascular stent-graft in abdonimal aortic aneurysm repair (EVAR)
}

\author{
Filippo Micheletti $^{1^{*}}$, Francesca Rossi ${ }^{1}$, Sara Condino ${ }^{4}$, Vincenzo Ferrari ${ }^{3,4,}$, Roberta Piazza ${ }^{2,3,4}$, Roberto \\ Pini $^{1}$, Mauro Ferrari ${ }^{2,4}$ \\ 1 IFAC-CNR, Institute of Applied Physics "Nello Carrara”, Italian National Research Council, Florence, Italy \\ 2 Vascular Surgery Unit, Cisanello University Hospital AOUP, Pisa, Italy \\ 3 Information Engineering Department, University of Pisa, Pisa, Italy \\ 4 EndoCAS, Department of Translational Research and of New Surgical and Medical Technol-ogies, University of Pisa, Pisa, \\ Italy \\ *f.micheletti@ifac.cnr.it
}

\begin{abstract}
Endovascular abdominal aortic aneurysms repair (EVAR) is a technique which involves the minimally invasive implantation of a stent-graft within the aorta to exclude the aneurysm from the circulation, preventing its rupture.

The feasibility of such operation is highly dependent on the aorta morphology and in general the presence of the visceral arteries (e.g. the renal arteries) emerging from the aneurysm is the absolute limit for the implantation of a standard stentgraft. Hence, classical intervention method involves the implantation of a custom-made graft with fenestrations, leading to the use of expensive prosthesys and a very complicated surgery with high risks for the patient.
\end{abstract}

More recent techniques introduced the use of standard grafts (i.e. without fenestrations) realizing a mechanical in-situ fenestration, but this procedure is limited principally by the brittleness and low stability of the environment. Also the difficult of guidance of the endovascular tools due to the temporarily block of blood flow towards the kidneys is a big limitation.

In this work an innovative EVAR procedure is proposed, which involves the in-situ fenestration of a standard stentgraft with a fiber guided laser tool, controlled via an electromagnetic navigation system.

Such a tool allows the surgeon to bring the laser directly inside to a previously released standard prosthesys using an endovascular instrument and there to selectively perforate its fabric avoiding damages to the aorta wall.

The proper fenestration site can be addressed with the support of the navigation system, which tracks the tip of the fiber displaying it in a 3D model of the patient anatomy.

The realization of the endovascular laser tool is presented, togheter with preliminary fenestration tests on graft-materials, including the effect of blood and tissues presence, and on the reliability of the electromagnetic sensors used to track the laser tool.

Keywords: EVAR, endovascular, laser

\section{Introduction}

When the abdominal aortic aneurism involves the region of the renal branches, in the endovascular approach, a fenestrated stent-graft must to be used in order to preserve the proper blood flood toward the kidneys [1].

Such prosthesis must be custom-made realized on the patient morphology [1],[2] and this requirement leads to several complication: first of all the device is quite expensive and it is not immediately available; then the surgery is quite challenging due to the fact that the prosthesis must be accurately released in order to match the fenestrations with the lumen of the renal branches.

A different approach has been proposed in literature, related to the implantation of a standard prosthesis (i.e. without fenestrations) with an in-situ mechanical fenestration [10], [11].

While this alternative procedure could lead to several advantages related to the costs and to the ease of release of the prosthesis, some big drawbacks are introduced. The most important is the high risk of rupture of the aneurysm during the perforation, due to the brittleness of such a tissue; then the possibility of a failure perforation, due to the low stability of the environment and to the impossibility of using the angiography to properly address the fenestration site, due to the temporary block of blood flow toward the kidneys, caused by the stent-graft itself [3],[4].

Both this problems can be overcome with the introduction of a different way to realize the perforation and to address the fenestration site.

In particular, taking advantage of the so called "transparency window" of biological tissues, we can use a laser emitting in the near infrared (NIR) wavelegth to selectively fenestrate the fabric of the prosthesis (made of DACRON, a particular polyester fiber) avoiding to damage the underlying aorta wall $[5],[6]$.

The laser light can be brought inside to the aneurysm using an optical fiber, which can be properly drived using an 
electromagnetic tracking togheter with a 3D model of the patient anatomy, in order to successfully address the fenestration site [7],[8].

\section{Materials and Methods}

A procedure for the laser approach to the in-situ fenestration has been defined in the following three steps:

- releasing of a standard stent-graft inside the aneurysm;

- driving a first endovascular laser tool up to the fenestration site and perform a first, small in diameter, perforation;

- drive a second endovascular tool on the realized fenestration and enlarge its diameter in order to restore the blood flow toward the kidney.

The need of a two-step fenestration is due to the fact that the optical fiber which brings the laser light must be vertically oriented respect to the prosthesys fabric, in order to reliably perforate it. This requirement leads to the need of bending the optical fiber brought there with a specific endovascular tool, and this set a limitation to the maximum diameter of the fiber that can be used [8]. Furthermore doing a small hole requires a relatively small amount of power which is preferable in this application respect to an higher one.

An endovascular laser tool has been so realized. It is obtained starting from a nitinol tube used as a guidwire for several endovascular standard tool, which has been equipped with the optical fiber and a sensor for the electromagnetic tracking system.

The Figure 1 shows the realized tool, where the connectors for the laser and the sensor are clearly visible, while Figure 2 shows the parts of the sensor.

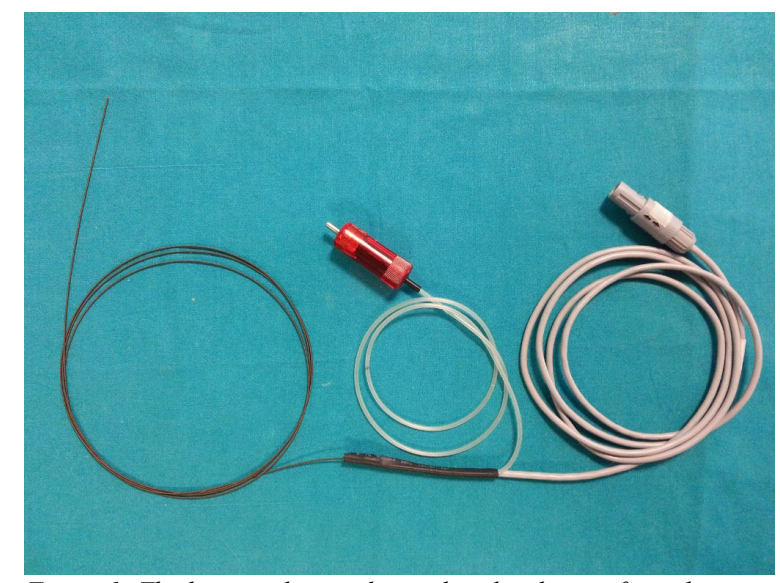

Figure 1: The laser endovascular tool realized to perform the fenestration.

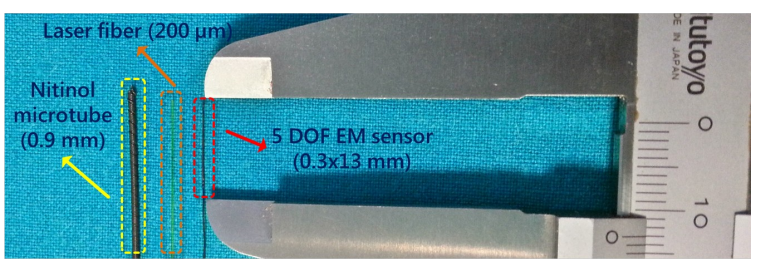

Figure 2: Parts of the realized sensor.

The used optical fiber is a 0.22 numerical aperture, high power, multimode one, with a $200 \mu \mathrm{m}$ core diameter, produced by THORLABS Inc, Newton, New Jersey.

The used laser is a commercial device for dentistry applications produced by DEKA, Calenzano, Italy, with a diode source emitting up to $10 \mathrm{~W}$ at a wavelength of $810 \mathrm{~nm}$.

The navigation system is based on the Northern Digital Inc Waterloo - Canada Aurora electromagnetic tracking system which is able to track 5 degrees of freedom coils with an accuracy of $0.7 \mathrm{~mm}$ in position and $0.2^{\circ}$ in orientation. The navigation system global positioning error of $1.2 \pm 0.3 \mathrm{~mm}$ (as resulted from a previous vitro study [8]) can be considered enough for such application.

\section{Results}

The built laser tool has been tested in order to verify its ability to perforate the DACRON fabric of the endovascular prosthesis and the reliability of the driving system.

For this purpose a piece of DACRON has been prepared on a plastic support realized in order to be easily submerged with human blood. So the laser tool has been placed in a support connected to a vertical micropositioner, in order to be able to accurately set the position of the fiber tip respect to the fabric surface, inside the blood. Figure 3 shows the setup.

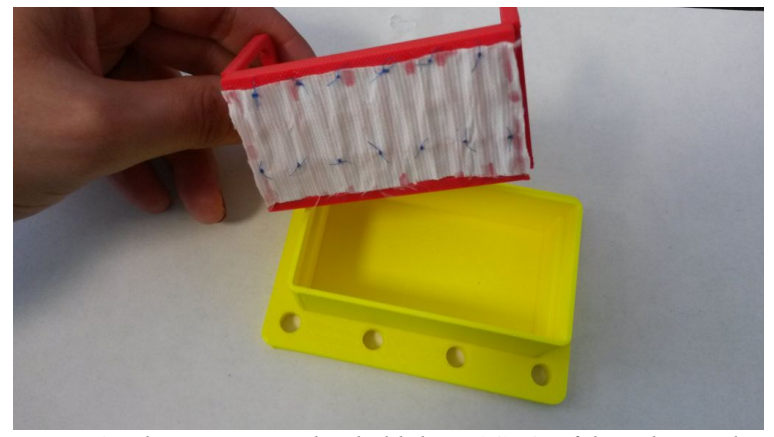

Figure 3: The support used to hold the DACRON fabric during the perforation test.

Several perforation trials has been conducted, varying the laser power between 0 up to $4 \mathrm{~W}$, the irradiation time in 0.5 and $0.9 \mathrm{~s}$, and the distance between the fiber tip and the fabric surface from 0 up to $3 \mathrm{~mm}$ [9].

An emission power threshold for a safe perforation has been identified in a value of $3 \mathrm{~W}$, and an irradiation time of 0.5 
seconds; these conditions has been veryfied to be valid from the contact between the fiber tip and the fabric up to about 2 $\mathrm{mm}$ of distance between them, a realistic working condition [9].

Figure 4 a) and Figure 4 b) show the example of two trials, one successful and one not.

During the same experiment the reliability of the microcoil sensor used for the electromagnetic tracking system has been also verified in terms of accuracy of the detected position before and after the perforation. For this purpose a bunch of about a hundred of position measurements has been acquired both before and after the perforation, veryfing the accuracy in terms of mean value and deviation, obtaining the same results, confirming that sensors were not damaged during the perforation.

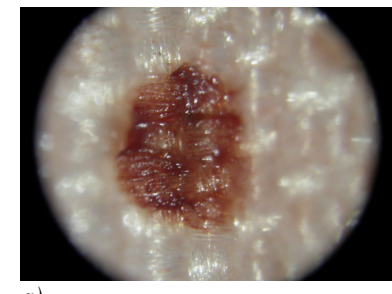

a)

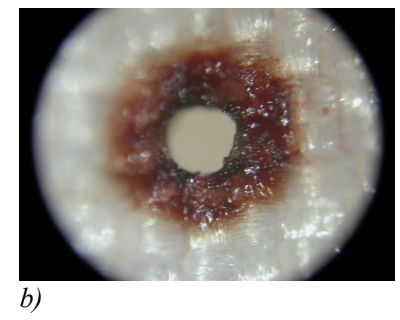

b)
Figure 4: Example of an unsuccessful perforation trial a), and a successful one b).

\section{Conclusions}

In this work the use of a laser tool in order to selectively fenestrate a standard prosthesis in the EVAR procedure has been demonstrated. The built laser endovascular tool has demonstrated to be able to perforate the DACRON in presence of blood for a reasonable setting suitable with the endovascular surgery. Esperiments has allowed also to define the minimum operative conditions required to safely obtain the perforation, that can be intended as a sort of perforation threshold. It has been veryfied also that the sensor of the tracking system is not damaged during the perforation.

Some further tests on the perforation of the prosthesis fabric are currently in progress, including the use of human ex-vivo tissues of the aorta wall, intended to prove the lack of unwanted effects due to the perforation.

Moreover the design of a second endovascular tool with the purpose of enlarging the fenestration diameter is in progress.

\section{References}

[1] Dillavou E.D., Muluk S.C., Rhee R.Y., Tzeng E., Woody J.D., Gupta N., Makaroun M.S, "Does hostile neck anatomy preclude successful endovascular aortic aneurysm repair?", Journal of vascular surgery 38, 657663 (2003)
[2] Cameron J.L., Cameron A.M., "Current Surgical Therapy", Elsevier Health Sciences (2013)

[3] Verhoeven E.L., Zeebregts C.J., Kapma M.R., Tielliu I.F., Prins T.R., Van Den Dungen J.J., "Fenestrated and branched endovascular techniques for thoraco-abdominal aneurysm repair", Journal of Cardiovascolar Surgery 46, 131-140 (2005)

[4] Greenberg R.K., Qureshi M., "Fenestrated and branched devices in the pipeline", Journal of vascular surgery:official publication, the Society for Vascular Surgery [and] International Society for Cardiovascular Surgery, North American Chapter 52, 15S-21S (2010)

[5] Murphy E.H., Dimaio J.M., Dean W., Jessen M.E., Arko F.R., "Endovascular repair of acute traumatic thoracic aortic transection with laser-assisted in-situ fenestration of a stent-graft covering the left subclavian artery", Journal of endovascular therapy : an official journal of the International Society of Endovascular Specialists 16, 457-463 (2009)

[6] Topaz O., "Lasers in Cardiovascular Interventions", Springer London (2015)

[7] Condino S., Ferrari V., Freschi C., Alberti A., Berchiolli R., Mosca F., Ferrari M., "Electromagnetic navigation platform for endovascular surgery: how to develop sensorized catheters and guidewires. The international journal of medical robotics + computer assisted surgery" MRCAS 8, 300-310 (2012)

[8] Condino S., Piazza R., Micheletti F., Rossi F., Pini R., Ferrari V. and Ferrari M., "Electromagnetic Guided InSitu Laser Fenestration of Endovascular Stent-Graft: endovascular tools sensorization strategy and preliminary laser testing", Medical Imaging and Augmented Reality (2016)

[9] Micheletti F., Pini R., Piazza R., Ferrari V., Condino S. and Rossi F., "In-situ laser fenestration of endovascular stent-graft in abdominal aortic aneurysm repair (EVAR)", Diagnostic and Therapeutic Applications of Light in Cardiology, Proc. SPIE 10042, 100420D (2017)

[10] Bismuth J., Duran C., Hassoun H.T.: In situ fenestration for branch vessel preservation during EVAR. Methodist DeBakey cardiovascular journal 8, 33-36 (2012)

[11] Saari P., Lahteenvuo M., Honkonen K., Manninen H.: Antegrade in situ fenestration of aortic stent graft: invivo experiments using a pig model. Acta Radiol 53, 754758 (2012) 